\title{
Low Dose Electron Ptychography for Cryo-biological Imaging
}

Liqi Zhou ${ }^{1}$, Jingdong Song ${ }^{2}$, Judy Kim ${ }^{3}$, Xudong Pei ${ }^{1}$, Chen Huang ${ }^{4}$, Xiaoqing Pan ${ }^{5}$, Peter Nellist ${ }^{4}$, Peijun Zhang ${ }^{4}$, Angus Kirkland ${ }^{4}$ and Peng Wang ${ }^{1}$

${ }^{1}$ Nanjing University, Nanjing, Jiangsu, China (People's Republic), ${ }^{2}$ Chinese Center for Disease Control and Prevention, Beijing, Beijing, China (People's Republic), ${ }^{3}$ Electron Physical Science Imaging Centre, Diamond Light Source, Didcot, England, United Kingdom, ${ }^{4}$ University of Oxford, Oxford, England, United Kingdom, ${ }^{5}$ University of California - Irvine, Irvine, California, United States

Cryo electron microscopy (cryo-EM), as one of the main technology for structural biology, has been attracted continuous efforts to develop techniques for the structure analysis of biological samples in a closer-to-native state at high resolution, such as single particle analysis [1]. As biological materials are very sensitive to radiation damage by an electron beam, the exposure to beam needs to be largely minimized, which, in turn results in each individual image with low-contrast and a poor signal-to-noise ratio. By defocusing the objective lens, the phase contrast can be largely improved. Nevertheless, defocusing the image corrupts the information transfer at intermediate and high spatial frequency[2]. Therefore, it is important to devise ways to improve resolution and contrast without damaging samples, including direct electron detectors, novel phase plates. One efficient way is to add a phase plate, like Volta phase plate [3] in an electron microscope. However, the routine applications of the phase plate are still restricted by the poor reliability and stability yet.

Alternatively, electron ptychography has gained the great interest for its unique potential in superresolution imaging [4, $\underline{5}$ ], high-contrast light-element detecting []], low dose imaging [7] and threedimensional imaging [요무. Especially, the advent of advanced direct electron detectors dramatically increase the signal-to-noise ratio and heightens the dose efficiency of ptychography [7]. Given its high resolution, high phase-sensitivity, high dose efficiency, and 3D imaging capability, electron ptychography has a great potential for low dose biological imaging with high contrast.

Here, we experimentally demonstrated low-dose ptychography with a defocused probe for biological imaging at both room temperature and cryo-state. A $1.14 \times 1.14 \mu \mathrm{m}$ wide area of an Adenovirus infected cell embedded in resin was raster-scanned with a defocused probe in the room temperature as shown in Fig. 1 (a). Fig. 1 (b) shows the ptychographic reconstructed phase image of the adenovirus-infected cell at the dose of $27 \mathrm{e} \AA^{2}$. The ptychographic phase shows good visibility of ultrastructural features of varied size, including cytoskeletal elements, viral particles, vacant vesicles, transport vesicles and free ribosomes as shown in Fig. 2. As already noted, the reconstruction of the ptychographic phase preserves low spatial frequency information, facilitating the recovery of these key molecular features. Since ptychography can provide high phase contrast for viral particles and on a large area of micro-meter scale with high dose efficiency, our further experiments at the liquid $\mathrm{N}_{2}$ temperature show that ptychography is a promising candidate for cryo-EM biological imaging. Moreover, this technique potentially paves a way to determine the 3D structure of biological samples in conjunction with cryo-EM single particle analysis and cryo-EM tilt series tomography [10]. 

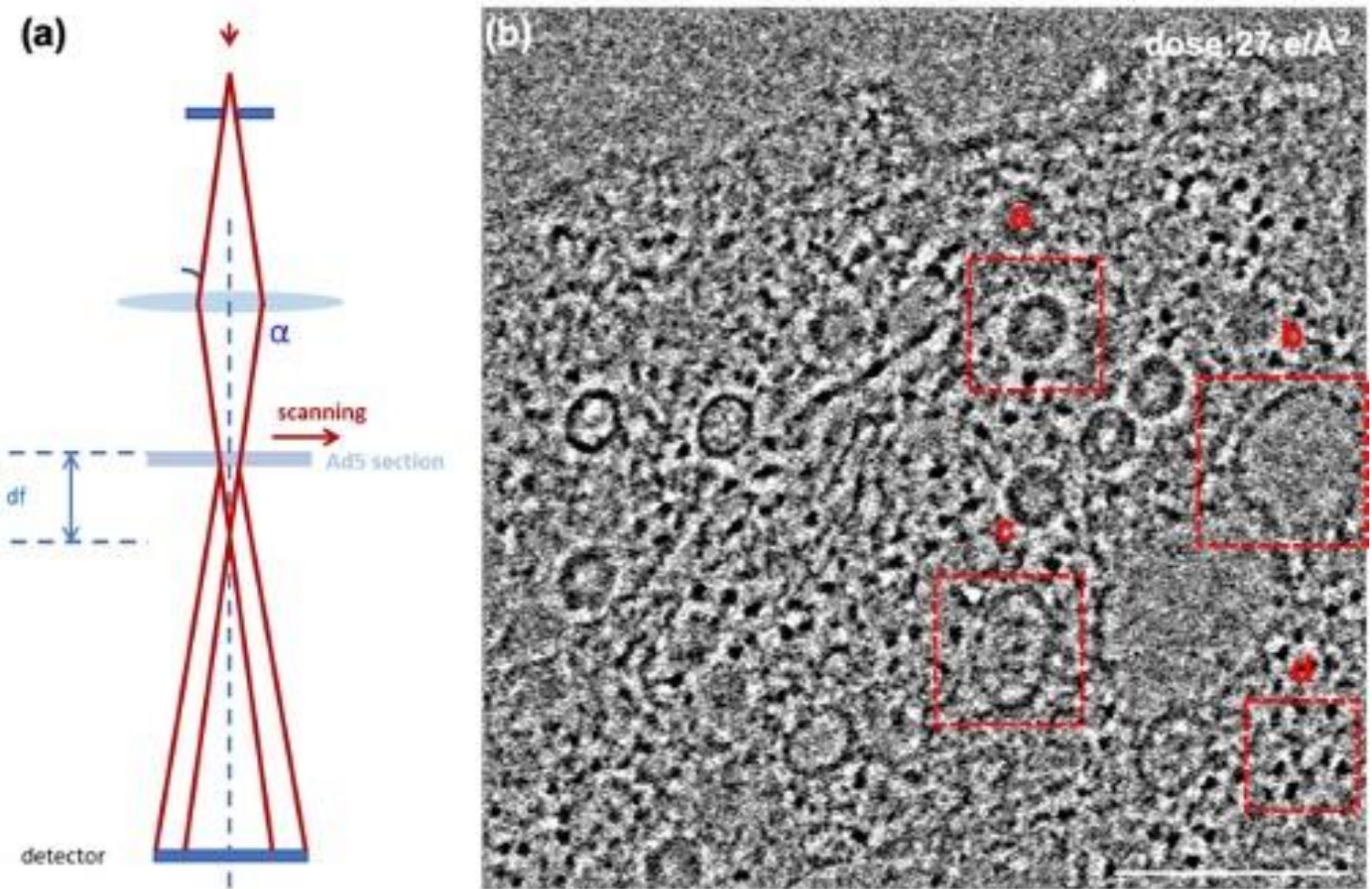

Figure 1. (a) The optical configuration schematic diagram. (b) Micrometer scale reconstruction of an adenovirus-infected cell at a dose of $27 \mathrm{e} / \AA 2$. Scale bar in (b) is $300 \mathrm{~nm}$,
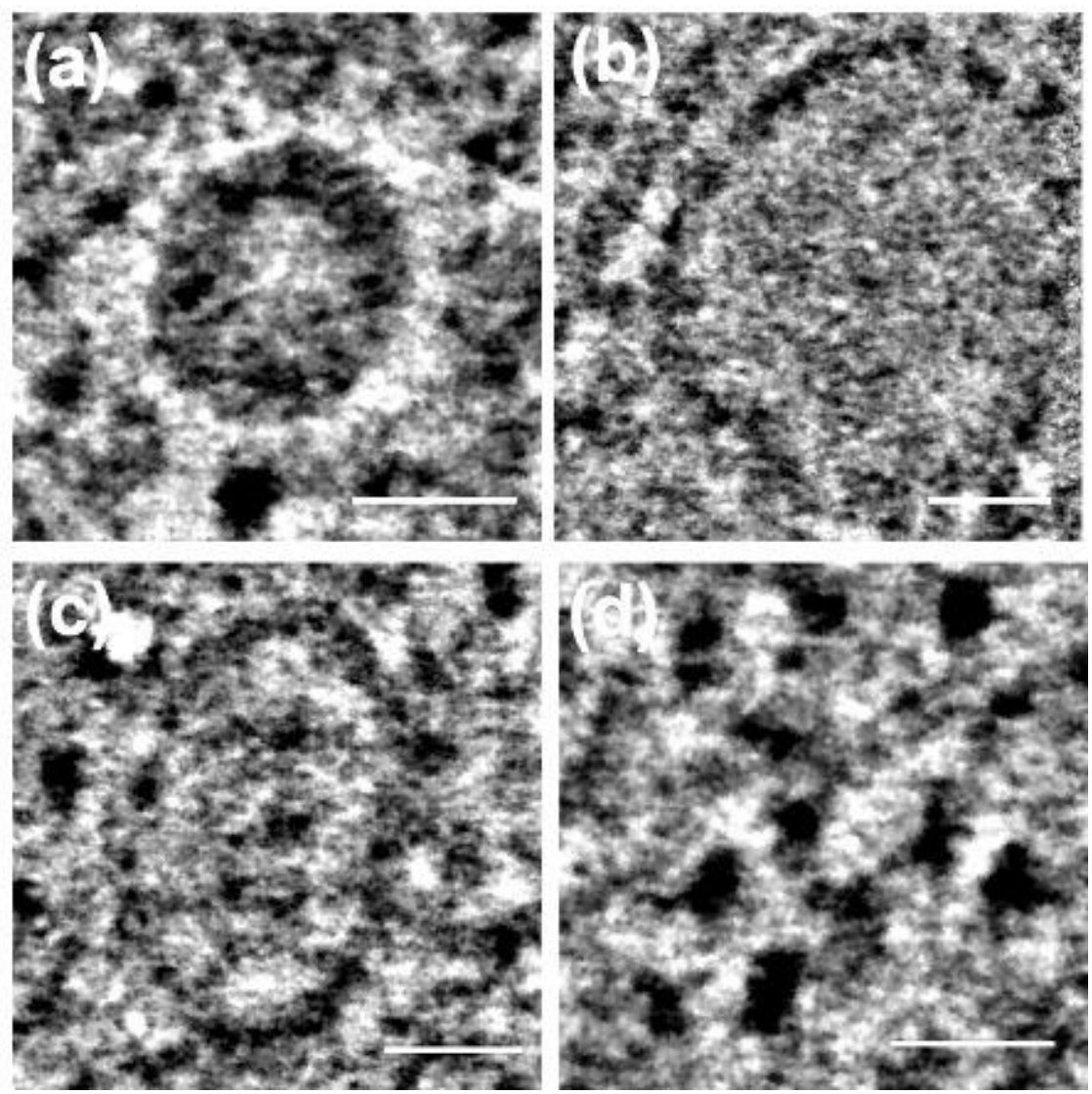
Figure 2. Magnified views of (a) a viral particle, (b) a vacant vesicle and (c) a transport vesicle from regions indicated with red squares in Fig 1 (b). And the small dots with high contrast in (d) are free ribosomes. Scale bars are $50 \mathrm{~nm}$.

\section{References}

[1] R. Henderson et al., Journal of Molecular Biology 213, 899 (1990).

[2] K. H. Downing et al., Ultramicroscopy 108, 921 (2008).

[3] R. Danev et al., Proceedings of the National Academy of Sciences 111, 15635 (2014).

[4] P. D. Nellist et al., Nature 374, 630 (1995).

[5] Y. Jiang et al., Nature 559, 343 (2018).

[6] P. Wang et al., Scientific Reports 7, 2857 (2017).

[7] J. Song et al., Scientific Reports 9, 3919 (2019).

[8] A. M. Maiden et al., J. Opt. Soc. Am. A 29, 1606 (2012).

[9] S. Gao et al., Nature Communications 8, 163 (2017).

[10] The authors acknowledge funding from the National Natural Science Foundation of China (11874199). 